\title{
Improving the quality of discharge summaries for elective surgical procedures at North Bristol NHS Trust
}

\author{
Emma Ladds, Frederica Betteridge, Sarah Yamamoto, Thomas Gupta-Jessop \\ NBT NHS
}

\begin{abstract}
Following elective surgical procedures, hospital discharge summaries are essential in the handover to primary care. The information provided varies between institutions and is highly user-dependent. Various interventions have focused on improving information transfer to patients and primary care physicians including the development of electronic templates, electronic transmission to primary care, and training initiatives for junior doctors.
\end{abstract}

An evaluation of the urological patient's journey at Southmead Hospital revealed a need for improved discharge summary advice. Urology specialists developed "gold standard" templates for elective urological procedures. Following a new rotation of junior doctors, discharge summaries were audited for one week. The templates were then made available on the urology ward, a teaching session was employed to encourage compliance, and the hospital electronic discharge summary template was edited. Following each intervention, summaries for one week of urology procedures were audited to assess the quality of advice provided to patients.

At baseline, $18 \%$ of discharge summaries contained sufficient patient advice, this reduced to $10 \%$ after templates were made available on the wards, increasing to $45 \%$ following the education session and $84 \%$ once the electronic discharge summary proforma was edited. We conclude that discharge summaries are an effective time point for intervening to provide patients with specific post-operative information and this may be optimised for different elective procedures via the introduction of electronically-distributed standardised templates.

\section{Problem}

It has been recognised nationally that discharge summaries following elective surgical procedures are not providing adequate information for individuals, and the Comptroller and Audit General Special Report in 2014 noted that less than a third of patients were being given appropriate written information on discharge. The report recommended that as a minimum patients should be given: 'appropriate [written] information about essential post-discharge issues, including emergency contact details and pain relief guidance.' (1).

"Advice to patient" and "advice to GP" are optional elements on the local eDischarge template, which are particularly poorly completed by junior doctors. This results in a heightened insecurity among patients on discharge; they have a lack of knowledge about possible complications to look out for and how to deal with them, or the most appropriate mechanism for seeking help. Similarly, GPs may be unaware of the required follow-up for their patients, which may result in missed or incorrect post-operative treatment. 'Finally, inefficient use of services may ensue, due to patient insecurity and help-seeking from inappropriate sources such as A\&E (2), whereas the evidence has shown no benefit in outcomes to hospital presentation for the majority of individuals recovering from elective surgery compared to being cared for effectively in the community (3).'

'An analysis of the Urological Patient's Journey at Southmead Hospital revealed that there were numerous points at which more information could be provided regarding appropriate post-operative care: at the initial diagnostic/therapeutic planning clinic appointment or follow-up sessions; pre-operative assessment; on the day of surgery; during the inpatient recovery period or on discharge. It was felt that the urology service was not maximising the opportunity of this latter timepoint for provision of written information for the patient, both in terms of opportunity and patient mindset.'

\section{Background}

Electronic discharge summaries are becoming increasingly popular as a method of post-discharge communication with GPs and patients, whether sent electronically or completed online and then printed and given to patients. Recent projects include development of and electronic discharge summary for a trauma and orthopaedic service [4] and increasing the proportion of discharge summaries transmitted electronically to GPs. [5] These electronic summaries have specific templates with required and optional fields for completion. Some services have developed specific templates with the minimal information required for particular procedures. For example, a stroke service recently developed a stroke-specific discharge summary template [6] and a pacemaker insertion service introduced specific guidelines on the minimum amount of information about the pacemakers required for inclusion on the summary.[7] Development of procedure-specific discharge summary templates may be effective at improving the quality of information transmitted to patients and GPs following elective surgical procedures. 
'The importance of such interventions has been recognised nationally in the United Kingdom and The Royal College of Physicians has provided guidance for Trusts on the use of electronic discharge summaries, including a toolkit for how to implement such initiatives (8).

\section{Baseline measurement}

Given the large number of elective procedures performed, it was decided to focus on one particular service to monitor the discharge summary intervention. Due to the large number of standard procedures carried out, the uro-oncological service was chosen. Following the start of a new rotation of junior doctors, the number of electronic discharge summaries with the "patient advice" sections completed for one week of procedures were measured.

This involved collating the total uro-oncology procedures performed in one week using only their unique hospital numbers to aid with patient anonymisation. The two junior doctors working in the uroology service compiled this list and distributed the MRN numbers amongst other members of the QI team, who were responsible for following the patients up on discharge and analysing their discharge summary. The analysis was made as objective as possible. Individuals were required to state 'yes' or 'no' twice, depending on whether the 'advice to patient' and 'advice to GP' contained any relevant information at all. Incorrect information was regarded as a 'no', but entries were not required (at baseline) to be comprehensive for all possible information in order to be ranked 'yes'.

It is possible that had the junior doctors compiling the list of MRN numbers also been involved in completing the discharge summaries themselves and performing the data analysis, they may have filled out these sections with more diligence than if they were blind to the intervention. Similarly this would have impacted on the further results collected following the introduced interventions, as these doctors would be more sensitive to correct discharge summary completion by virtue of being involved in the QI project. However, we attempted to control for this by completing our baseline measurement and introducing subsequent interventions at the start of a new rotation of junior doctors - thus hoping to minimize such bias.

\section{Design}

Our intervention involved designing discharge summary templates for specific uro-oncology procedures based on the advice of consultants and senior registrars.

Following initial baseline measurement of the number of electronic discharge summaries with 'advice to patients' completed with any relevant information, the initial intervention involved introducing these templates in hard format for a new rotation of junior doctors working in the urology service to use and re-auditing the discharge summaries over five days (as described in the baseline measurement section). Again we did not require the information provided to be $100 \%$ comprehensive, but incorrect advice was ranked as 'no' on the analysis.

The second intervention involved holding an informal teaching session with the doctors completing the summaries to encourage compliance; the third involved introduction of a compulsory section onto the electronic discharge summary template to ensure 'advice to patient' was provided and finally the discharge summary templates were uploaded onto the urology hard drive for easy accessibility.

Following each of those steps the summaries were re-audited.

It may have been possible to break the PDSAs down in terms of length of time audited. However, we were not initially sure how many urology procedures we would need to audit in order to provide meaningful numbers, nor how many such procedures would be performed each day/week. Due to time constraints it was impossible to do a pilot study which might have provided us with this data. Future work should aim to include such a step to minimize the risk of overcomplicating PDSA cycles.

There were no additional costs associated with the intervention, and whilst we did not directly measure efficiency for junior doctors, anecdotally this was reported. Further work should directly measure this. By altering the generic discharge summary template we aimed to enhance the sustainability of the intervention.

Further/future work could include a more qualitative approach to discussing with junior doctors the barriers they felt hindered their completion of discharge summaries and whether our interventions were helpful. We would also have liked to involve patients more directly. We did conduct an analysis of the urological patient's journey (see baseline measure) and attempted to discuss with urology patient support groups about the utility of our intervention, however, that proved impossible due to the time limitation of 6 months imposed on our project. Further work should focus on a deeper analysis of patient's views.

\section{Strategy}

PDSA 1: Gold standard template provided on urology ward: predicted increase but actually decrease from $18 \%$ (at baseline) to $10 \%$ of 'advice to patient' sections completed on uro-oncology discharge summaries

PDSA 2: Gold standard template provided on urology ward and informal education session for urology junior doctors: predicted increase and did see increase of $10 \%$ to $45 \%$ of 'advice to patient' sections completed on uro-oncology discharge summaries

PDSA 3: Trust e-discharge proforma edited to make 'advice to patients' essential and urology templates made available on Trust hard drive: predicted increase and saw increase to $84 \%$.

We had expected to perform 4 PDSA cycles, but were forced to amalgamate the last two (trust e-proforma edited and templates on hard drive) due to the IT department changing the template ahead of schedule. 
See supplementary file: ds4723.pdf - "Run Chart"

\section{Post-measurement}

Patients said that they did not receive adequate advice about the practicalities of post-operative care, there was little objective evidence to support an absence of advice on hospital discharge summaries.

Data was collected from one week of discharge summaries for a selection of uro-oncology procedures performed by the urology department in a single tertiary referral centre. The percentage of "advice to patient" sections were filled in on the electronic discharge summaries, with notes made for incorrect or absent information compared to the gold standard templates.

Baseline: $18 \%$

Initial Intervention: Distribution of discharge summary templates to urology ward: $10 \%$

2nd Intervention: Informal educational initiative for junior doctors: $45 \%$

3rd Intervention: Compulsory e-discharge summary "advice to patients" section and distribution of the discharge summary templates onto the junior doctors' shared hard drive: $84 \%$

\section{Lessons and limitations}

We learned a number of lessons during this project. Initially the enthusiasm and support of senior urology doctors involved in designing the templates and providing advice was significant, but this waned as the project progressed. It was crucial to contact all the individuals involved in the production of the discharge summaries, ensuring they bought into the importance of providing advice for patients in this manner. It may be that there are alternative or additional methods of providing helpful, adequate advice for patients and their relatives alongside discharge summaries. However, unless the junior doctors and nurses appreciated the importance of the discharge summaries themselves as a brief, succinct reference for patients and their families, then they viewed the project as a tick-box exercise rather than having any intrinsic merit. There were also small practical difficulties encountered. The doctors carrying out the project are split over two hospital sites and as such co-ordination of tasks and continuity was challenging. Distribution of forms on the wards was complicated by the fact that this was just one more piece of paper and was easily misplaced. This was improved by making the template available electronically, which also allowed doctors to remotely complete discharge summaries more fully.

It was interesting that the percentage of completed sections actually reduced following the initial intervention, but increased following the second. This highlights the importance of targeting specific individuals (or increasing awareness among a group of specific individuals) before they are instigated. While the use of the templates reduced the amount of time required to complete the discharge summaries, there is initially an increase in time-outlay as it is necessary to locate the templates and then to cross-reference, etc.

\section{Conclusion}

Analysis of the urological patient's journey along with anecdotal information from colleagues revealed a lack of patient-centred postoperative information. Numerous time points were identified where interventions could be made, although we focused on the final discharge summary in this project. We demonstrated that the creation of a standard procedure-specific template could be used to improve the information provided for patients after elective urological procedures. These were most effectively distributed via the Trust electronic hard drives, although education initiatives for junior doctors and ward-based hard copies also improved discharge summary outputs.

Given the demonstration of an improvement in patient information following this simple intervention, there is clearly scope to expand it further. Future work should focus on ensuring sustainability (hopefully improved by the use of electronic templates) and developing new templates for additional elective procedures across different specialties.

\section{References}

1. Comptroller and Auditor General Special Report (2014) Managing Elective Day Surgery (downloaded 9/12/14 from http://www.audgen.gov.ie/documents/vfmreports/83_Electiv eDaySurgery.pdf)

2. Walley, P. (2004) Reducing Attendances and Waits in Emergency Departments. A systematic review of present innovations (downloaded on 9/12/14 from http://www.sdo.nihr.ac.uk/files/project/29-finalreport.pdf)

3. Shepperd, S. and lliffe, S. (2004) Hospital at home versus inpatient hospital care (Cochrane Review), in The Cochrane Library, Issue 3. Chichester, UK: John Wiley \& Sons, Ltd.

4. Evans, J, Armstrong, A. From Zero to Hero, the rise of the Trauma and Orthopaedic discharge summary. BMJ Qual Improv Report 2013;2: doi:10.1136/bmjquality.u201983.w1029.

5. Barr, R, Chin, KY, Yeong, K. Improving transmission rates of electronic discharge summaries to GPs. BMJ Qual Improv Report 2013;2: doi:10.1136/bmjquality.u756.w1013.

6. Mäkelä, P, Haynes, C, Holt, K, Kar, A. Written medical discharge communication from an acute stroke service: a project to improve content through development of a structured stroke-specific template. BMJ Qual Improv Report 2013;2: doi:10.1136/bmjquality.u202037.w1095.

7. Taylor, C. Improving e-discharge letters for Permanent pacemaker insertions at Wansbeck General Hospital. BMJ Qual Improv Report 2013;2: doi:10.1136/bmjquality.u201251.w772.

8. Royal College of Physicians (2011) New toolkit based on RCP discharge summary record launched (downloaded on 
9/12/14 from https://www.rcplondon.ac.uk/press-releases/ne w-toolkit-based-rcp-discharge-summary-record-launched)

\section{Declaration of interests}

None declared

\section{Acknowledgements}

The authors would like to acknowledge: North Bristol NHS Trust and specifically the urology department at Southmead Hospital. 